\title{
Analysis of Mathematical Models and Influence of Constructions of High Efficient Heat Transfer Devices
}

\author{
Alijon Abdiraupovich Kholikov, Hamrokul Kuvandikovich Sayidov, \\ Murodjon Hamidjonovich Beshimov
}

Bukhara Engineering-Technological Institute, Bukhara, Republic of Uzbekistan

Email address:

alexes76@bk.ru (A. A. Kholikov), saidovhk@gmail.com (H. K. Sayidov), alexes76@bk.ru (M. H. Beshimov)

\section{To cite this article:}

Alijon Abdiraupovich Kholikov, Hamrokul Kuvandikovich Sayidov, Murodjon Hamidjonovich Beshimov. Analysis of Mathematical Models and Influence of Constructions of High Efficient Heat Transfer Devices. Mathematical Modelling and Applications.

Vol. 2, No. 6, 2017, pp. 63-67. doi: 10.11648/j.mma.20170206.12

Received: March 20, 2017; Accepted: November 11, 2017; Published: December 13, 2017

\begin{abstract}
According to the research on mathematical model of the influence of constructions of porosity of the wick depending on diameter of the wires and impact of porosity, as well as factors such as distilled water and alcohol on coefficient of thermal conductivity, on coefficient of heat transfer devices. The conducted analysis of the parameters of influence of constructions of high efficient heat transfer device (HEHTD or thermal pipes) on power of density of the heat flow. The carried out calculations are necessary for making average temperature heat transfer devices.
\end{abstract}

Keywords: The Heat Pipe, Porosity, Thermal Conductivity, Water, Wick

\section{Introduction}

The process of drying fruit in the food industry is considered one of the most important processes. In the world market, dried products are in high and special demand for their quality properties - the level of vitamins preservation, color, shelf life, saturation with fructose and sucrose, medicinal properties.

To date, during the growing of fruits, their storage and processing before delivery to the consumer, about $20-30 \%$ of production is lost, which significantly affects the total value of entrepreneurs' income.

On these issues, studies were conducted on the possible extension of the storage period of fruit and vegetables for 1-2 years and, along with this, studies are conducted on improving the quality, environmental friendliness and ensuring natural storage conditions.

For the long-term storage of fruits, the technological process of drying plays an significant role. Drying is one of the improved processes, which depends on many factors. For example, the moisture content of the product, overall dimensions, the presence of solids, the specific heat of the product, and others. Taking this into account, the existing drying equipment of the food industry for drying fruit was studied and an improved laboratory model for drying fruit was developed.

On the basis of these studies, a drying plant has been created by using a highly efficient heat transfer device, where the highly efficient heat transfer device operates at the expense of solar energy and in which design can be distinguished: simplicity of design, exceptional maneuverability, ease of regulation and the possibility of transferring high heat fluxes over a considerable distance with extremely low temperature pressure $[1,2]$.

Energy saving processes, its development and implantation of highly effective engineering and technologies are indeed actual problems in enhancement of small and medium business entities.

Energy expenses on implementation of heat and mass transfer processes in the primary processing of raw materials and in the manufacture of food products make up $70 \%$ of the total energy used. Solar energy and wasted heat on the mass heat processes, and absence of applying high efficient heat transfer devices which are heat pipes (HP) are not adequately effective.

Thereby, one of the main objectives is to elaborate utilization of solar energy and wasted heat in heat mass transfer processes the employment of the highly effective heat-transmitting devices in different production equipment $[7,8,9]$. 
A heat pipe (HP), a heat transfer device capable of transmitting large thermal energy at small temperature gradients. HPs are hermetically sealed construction (pipe) partially filled with a heat liquid carrier. In the heated part of the HP (in the heating or evaporation zone), the heat transfer fluid evaporates with absorption of heat, and in the cooled part of the HP (in the cooling or condensation zone), the vapor flowing from the evaporation zone condenses with the release of heat. The movement of steam from the evaporation zone to the condensation zone is due to the difference in pressure of the saturated vapor, determined by the temperature difference in the evaporation and condensation zones. The liquid is returned to the evaporation zone either due to external influences (for example, gravity), or under the action of a capillary pressure difference along the capillary structure (wick) located inside the HP (most often on its walls). Due to the fact that HPs with a capillary structure for fluid return can work independently of orientation in the gravity field and in weightlessness, this type of HP is the most common [5].

The heat input can be carried out by any known method (open flame, heat exchange with a heated body, electric current, infrared rays, electron bombardment, etc.). In this case, due to the thermal resistance of the wall, there is a normal temperature difference between the heat source and the working fluid. The temperature limit depends only on the thermo- (cold) strength of the housing material.

Zone of evaporation. The density of the heat flux should not exceed a certain value, at which the heat transfer crisis may occur (draining of the wick, when does not catch up to feed new portions of liquid into the evaporation zone).

Condensation zone. There are two types of condensation a drop or, more often, a film. Usually, the vapor condenses on the surface of the liquid film. Between this surface and the outer wall surface there is a small temperature difference (i. e., the wall temperature is not much, but differs from the vapor temperature). The surface of the $\mathrm{HP}$ in the condensation zone has an interesting property isothermality, because this surface works practically at a constant temperature (close to the temperature of the vaporliquid phase transition). If a local heat flow occurs at some site, the amount of condensed steam in that place increases and the temperature is maintained at the same level.

Transport zone of steam. In the evaporation zone, an excessive vapor saturation pressure is created, which is expended to accelerate the steam to an axial velocity, to overcome the frictional force of the steam flow on the surface of the wick and to overcome the vapor viscosity forces. Because of this, the pressure along the length of the HP, from the evaporation zone drops. In the middle section of the pipe, the pressure stabilizes (adiabatic region). In the condensation zone, the pressure is restored to near the pressure in the evaporation zone. In practice, pressure losses in simple HPs are related only to the overcoming of viscous forces [4].

\section{Method}

Modern HPs have the following characteristics: operating temperature from $4 \mathrm{~K}$ to $2300 \mathrm{~K}$; Length from a few centimeters to tens of meters; Diameter from $2-3 \mathrm{~mm}$ to several meters; Heat transfer power up to $25 \mathrm{~kW} / \mathrm{cm}^{2}$; resource work up to 100000 hours. By the form of heat carriers, metal (potassium, sodium, cesium, etc.) is distinguished for very high temperatures and nonmetallic (water, ammonia, acetone, Freon (chlorofluorocarbons (CFCs), etc.) for low and medium temperatures. To bring the condensate back to the evaporation zone, gravity, capillary, centrifugal, electrostatic and etc. energies can be used. [6]

In the experimental laboratory installation, it is possible to determine the operability of the HP. The laboratory heat pipe consists of five main parts, as shown in Figure 1, namely: the body, the wick, the end cap, the fill tube and the coolant.

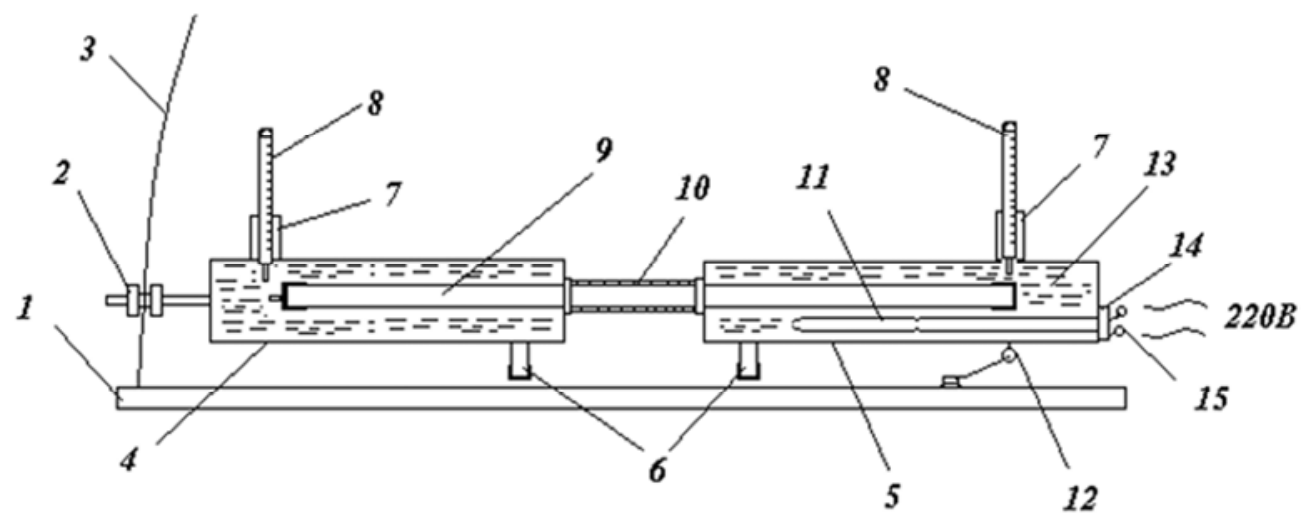

Figure 1. Test facility for medium temperature heat pipes.

1- frame; 2- fastening; 3- tripod; 4,5- body; 6- tubes for outflow; 7- downspout tube connections; 8- thermometers; 9- heat pipes; 10- thermal insulation; 11- a heating element; 12- hinge; 13- water; 14-thermo-regulating device; 15 -switch

The calculation of the porosity of thermal pipes can be computed from the formula - porosity of grid.

$$
\varepsilon=1-\frac{\pi \cdot S \cdot N \cdot d}{4}
$$

$S$ - bend of the wire $[1 \div 1,5]$,

$N$ - the number mesh,

$d$ - the diameter of wire, (in $m$ )

A study on the mathematical model of the influence of the 
diameter of the wire of reticulated material and from the number is mesh on the porosity of the wick it showed that, with an increase the number mesh decreases the porosity of wick.

For the analysis of the influence of material and porosity of reticulated wick, and also working agent on the coefficient of thermal conductivity, the algorithm of calculation has been developed.

The computed values of the coefficient of thermal conductivity depending on material and porosity of reticulated wick, and also of working agent it is executed according to the formula (2):

$$
\lambda_{p=} \frac{\lambda_{f}\left[\left(\lambda_{f}+\lambda_{m}\right)-(1-\varepsilon)\left(\left(\lambda_{f}-\lambda_{m}\right)\right]\right.}{\left[\left(\lambda_{f}+\lambda_{m}\right)+(1-\varepsilon)\left(\lambda_{f}-\lambda_{m}\right)\right]}, W t / m K
$$

$\lambda_{f}$ - the thermal conductivity of working agent,

$\lambda_{m}-$ the thermal conductivity of grid,

$\mathcal{E}$ - the porosity of grid, in the portions.

The results of calculating the dependence of the coefficient of thermal conductivity on material and porosity of reticulated wick, and also working agent are given in figure 2 .

The analysis of curves showed that, the coefficient of the thermal conductivity in the thermal pipes with the utilization of heat in mean temperatures with the use of a working agent of the distilled water it increases in the comparison of the working agent of alcohol $1.7-2$ and, with the increase of the porosity of reticulated wick from 0.6 to 0.84 (in the portions) the coefficient of thermal conductivity decreases within limits of $1.2-1.3$ times.

In connection with the influence of the porosity of reticulated wick on the coefficient of thermal conductivity it is necessary to consider its influence in the calculations of thermal pipes.

For the analysis of the influence of material and porosity of reticulated wick, and also working agent on the coefficient of thermal conductivity, the algorithm of calculation has been developed.

The results of calculating the dependence of the coefficient of thermal conductivity on material and porosity of reticulated wick, and also working agent are given in figures 2,3 .

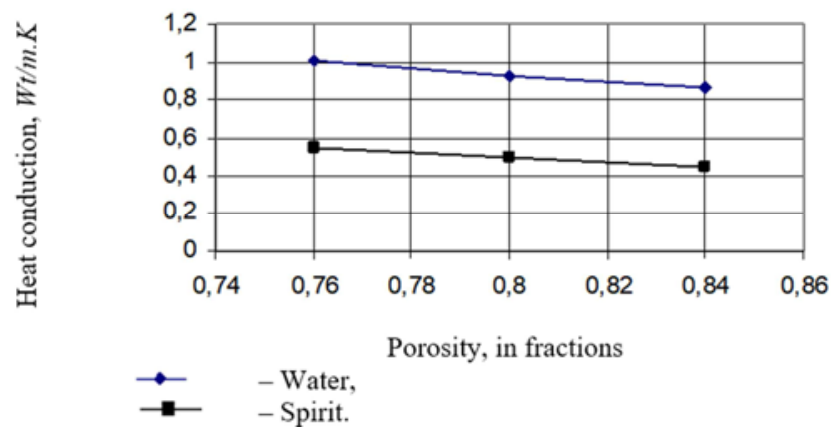

Figure 2. Dependence factor of conduction from various factors in a HP (Copper match $N=4800)$.

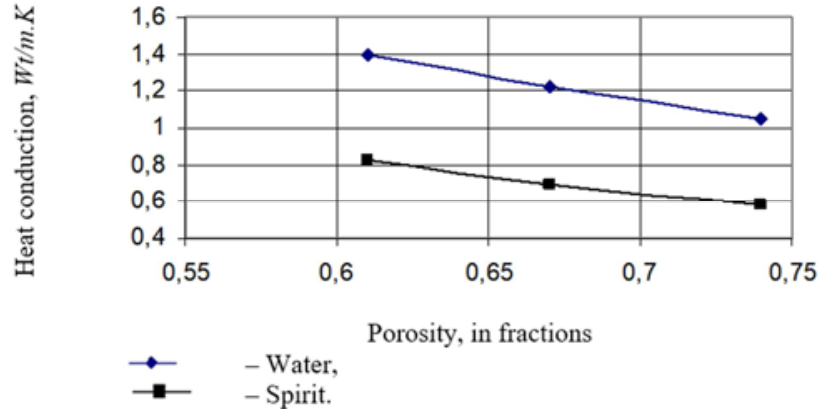

Figure 3. Dependence factor of conduction from various factors in a HP (Steel match $N=7800$ ).

The analysis of curves showed that, the coefficient of the thermal conductivity in the thermal pipes with the utilization of heat in mean temperatures with the use of a working agent of the distilled water it increases in the comparison of the working agent of alcohol $1.7-2$ and, with the increase of the porosity of reticulated wick from 0.6 to 0.84 (in the portions) the coefficient of thermal conductivity decreases within limits of $1.2-1.3$ times.

For the analysis of the influence of the characteristic of reticulated wick and working agent on the coefficient of the heat transfer, the algorithm of calculation has been developed.

The computed values of the coefficient of the heat transfer of the walls of thermal pipes it is executed according to the formula (3).

$$
\mathrm{K}=\frac{1}{\frac{1}{a_{1}}+\frac{1}{a_{2}}+\frac{\delta_{t}}{\lambda_{t}}+\frac{\delta_{p}}{\lambda_{p}}}, W t / m^{2} . K
$$

$\alpha_{1}$ - outside of heat irradiation of thermal pipe, $\mathrm{Wt} / \mathrm{m}^{2} . K$,

$\alpha_{2}$ - inside of heat irradiation of thermal pipe, $W t / \mathrm{m}^{2} . K$,

$\delta_{T p}$ - the thickness of the wall of thermal pipe, $m$,

$\lambda_{t}-$ the thermal conductivity of thermal pipe,

$\delta_{p}-$ thickness of wick,

$\lambda_{p}$ - thermal conductivity of wick.

The results of calculating the dependence of the coefficient of heat transfer in the condenser and evaporative part of the thermal pipes of the characteristic of reticulated wick and working agent are given in figures 4,5 .

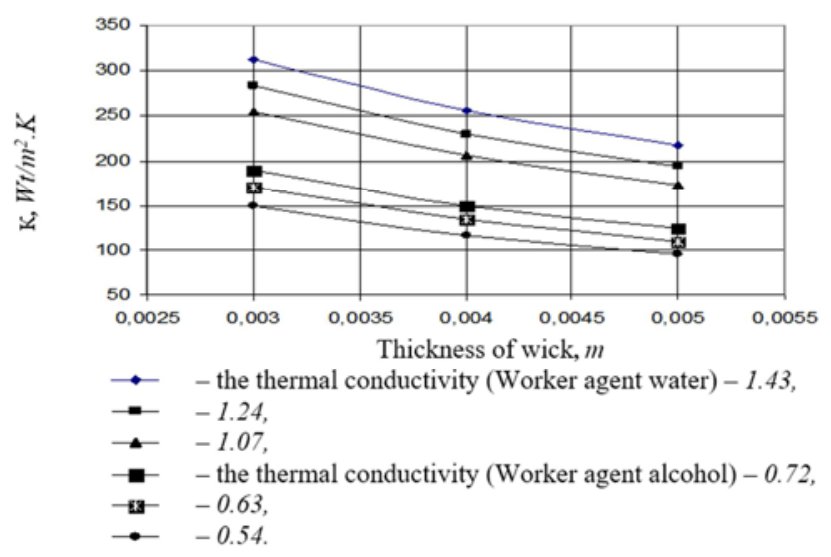

Figure 4. Dependences of the coefficient of heat transfer on thw thickness of wick in thermal pipes ( $N=7800$, Copper tube, copper wick). 


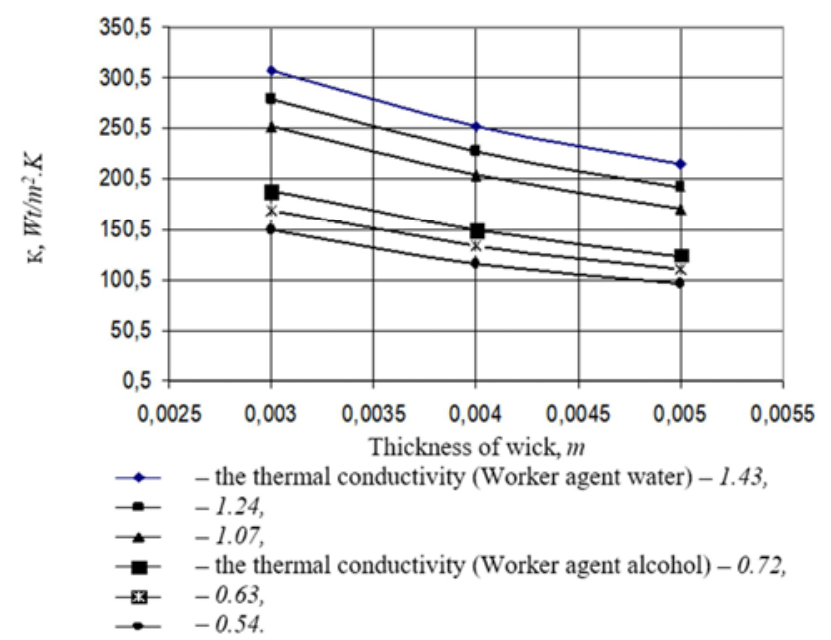

Figure 5. Dependence of the coefficient of heat transfer on the thickness of wick in thermal pipes ( $N=7800$, steel tube, copper wick).

Analysis of the curves of the coefficient of the heat transfer it showed that, with an increase in the thickness of the layer reticulated wick from 3 to $5 \mathrm{~mm}$ depending on material thermal pipes with the utilization of heat in mean temperatures $K$ decrease $1.3-1.4$ times.

For the analysis of the influence of the characteristic of reticulated wick, working agent and design sizes on the power of thermal pipes, the algorithm of calculation has been developed.

A study is the influence of different factors on the power of the thermal pipes it is produced according to the equation

$$
\mathrm{Q}=\mathrm{Fk} \Delta \mathrm{T}
$$

The results of investigating the influence of the factors of such as, material and the thickness of wick and also the material of working agent to the power of thermal pipes are given in figure 6,7 .

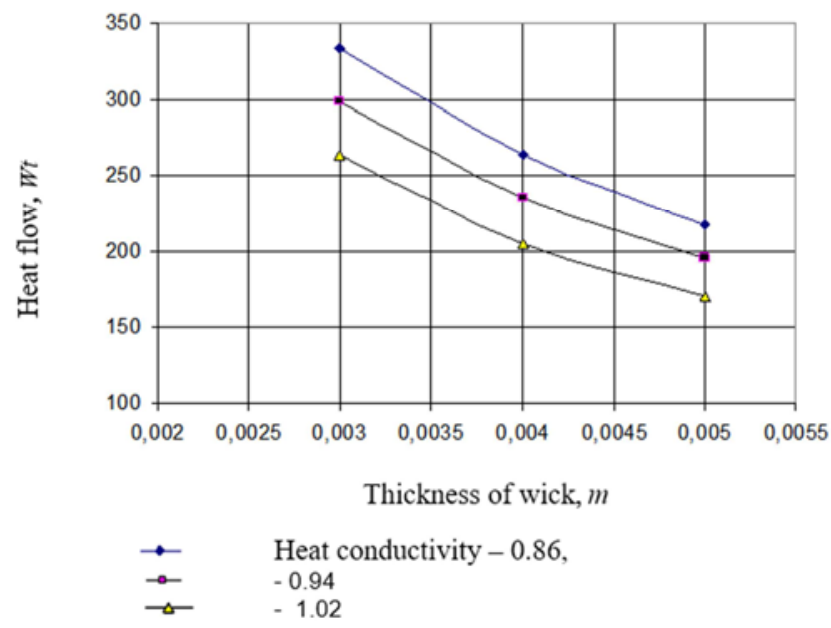

Figure 6. Dependency of the heat flow from different factor in thermal pipes.

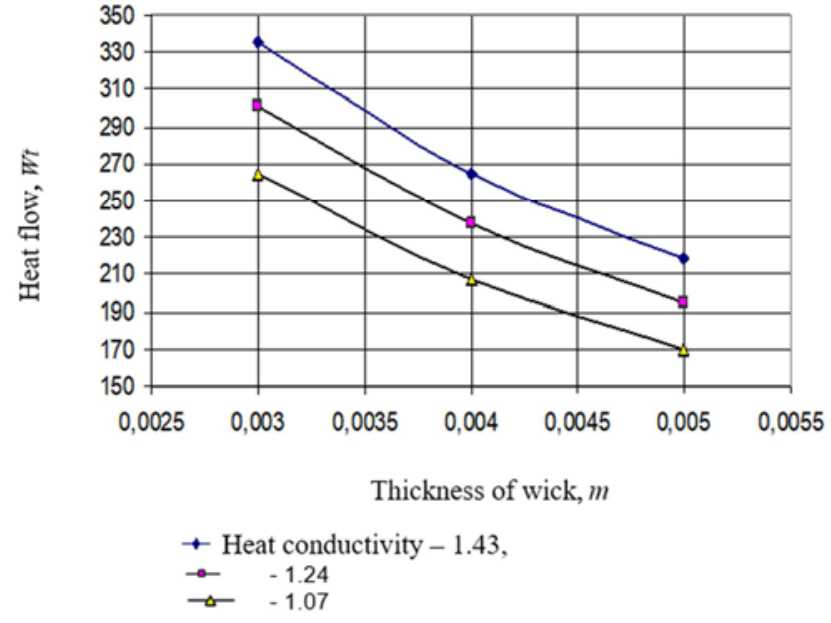

Figure 7. Dependency of the heat from different factor in thermal pipes.

Analysis of the results shows that, with the production of thermal pipes with the thickness of wick $3.0 \mathrm{~mm}$ with the copper material the power of heat flux to be $550 \mathrm{~W} /\left(\mathrm{m}^{2} \mathrm{j}\right)$ and, with the thickness of the layer of wick $4.5 \mathrm{~mm}$ the power of heat flux decreases to $380 \mathrm{~W} /\left(\mathrm{m}^{2} \mathrm{j}\right)$.

\section{Results}

Since the 1960s and 1970s, when it comes to the use of HP, spacecraft has been used for heating and cooling various devices, appliances.

Also, studies in this area were researched to the 80 's of the 20 th century and several new ideas were created. This article discusses the choice of HP corpus with heat transfer agent and wick, and experiments, for this particular process.

At the present time, coolers are used to cool down the central processors of computers, which can be called HPs. In some developed and hot climates, geothermal energy, such as the underground cold climate, is used by roadsides to keep the air condensation and maintain the air.

HPs are used in both agro-industry and solar energy, as well as for the use of solar energy and secondary derelict heat energy for low-temperature processes of the food produced in greenhouses and chemical industries. In particular, the Bukhara Engineering-Technological Institute has created a solar-powered HP-drier, and scientific researches are underway.

\section{Conclusions}

- Analysis of the results of research on the mathematical model shows that the development of heat pipes necessity depending on the source of the use of the application of the mesh wick with a volume of mesh 1dia. Sq. from 75 to 200 is effective;

- When using selected heat with an average temperature of the material chosen working fluid water, alcohol or ammonia, as well as recommend the use of the compositions by mixing them;

- Curve analysis showed that the heat transfer coefficient, 
increase in wick mesh layer thickness of 3 to $5 \mathrm{~mm}$ depending on the material of heat pipes with heat recovery in the medium temperature is decreased $K 1.3-1.4$ times;

- Analysis of the results shows that, when manufacturing heat pipes with a wick layer thickness of $3.0 \mathrm{~mm}$ made of copper heat capacity flow make $550 \mathrm{~W} /\left(\mathrm{m}^{2} \cdot \mathrm{K}\right)$ and, if the wick layer thickness is $4.5 \mathrm{~mm}$ then power of heat flow is reduced to $380 \mathrm{~W} /\left(\mathrm{m}^{2} \cdot \mathrm{K}\right)$ in connection with what is recommended in the manufacture of the heat pipes receive the wick thickness of 2 to $3 \mathrm{~mm}$.

\section{References}

[1] P. D. Dunn, D. A. Reay. Heat Pipes. Pergamon Press, Oxford, New York, Toronto, Sydney, Paris, Braunschweig, 1976. - 272 page.

[2] S. W. Chi. Heat Pipe Theory and Practice A Sourcebook. Hemisphere Pub. Corp. in Washington. 1976, 242 page.

[3] Stabnikov V. N., Lisyanskiy V. M., Popov V. D. Processes and machinery of production.:-M.: Agropromizdat, 1985 - 512 page.
[4] http://www.trizminsk.org/e/21102100.htm.

[5] http://engineeringsystems.ru/teploviye-trubi.

[6] Safarov A. F., Gafurov K. Kh., Khikmatov D. N., Kholikov A. A. Energy-saving technologies in the processing of fruits and vegetables. Bukhara. Publ: Durdona, 2013. -248 page.

[7] Ginzburg A. S. Fundamentals of the theory and technology of drying food products. - M.: Food industry. 1973. - 528 page.

[8] Ginzburg A. S. Calculation and design of plants of food drying industry. M.: 1985y. page.16.

[9] D. Reay, D. Macmichael. Heat pumps. Design and Applications. Pergamon press, Oxford, 1979. - 222 page.

[10] David P. DeWitt, Theodore L. Bergman, Frank P. Incropera, Adrienne S. Lavine Introduction to Heat Transfer 2011.

[11] E. R. G. Eckerst, R. M Drake - Analysis of heat transfer 1986.

[12] R. Rudramoorthy - Thermal Engineering 2017. 